\title{
Supernova Burst and Relic Neutrino Sensitivity Studies in the Hyper-Kamiokande Korean Sites
}

\section{Dong-Nyeok Yeum* and Seon-Hee Seo}

Department of Physics and astronomy, Seoul National University

E-mail: dnyeumesnu.ac.kr, shseodsnu.ac.kr

\section{Takatomi Yano and Yasuo Takeuchi}

Department of Physics, Kobe University

E-mail: tyano@phys.sci.kobe-u.ac.jp, takeuchi@phys.sci.kobe-u.ac.jp

\section{Motoyasu Ikeda}

Institute for Cosmic Ray Research, University of Tokyo

E-mail: motoyasu@suketto.icrr.u-tokyo.ac.jp

\section{Masashi Yokoyama}

Department of Physics, University of Tokyo

E-mail: masashi@phys.s.u-tokyo.ac.jp

\section{Itaru Shimizu}

Research Center for Neutrino Science, Tohoku University

E-mail: shimizu@awa.tohoku.ac.jp

\section{Yusuke Koshio}

Department of Physics, Okayama University

E-mail: koshio@okayama-u.ac.jp

\section{Jost Migenda}

Department of Physics and Astronomy, University of Sheffield

E-mail: jmigendalesheffield.ac.uk

\section{for the Hyper-Kamiokande Proto-Collaboration}

Hyper-Kamiokande is a next-generation water Cherencov detector for neutrino physics. Its large volume $(260$ kton $\times 2)$ allows Supernova burst $(\mathrm{SN})$ neutrino and Supernova Relic Neutrino (SRN) search much more promising than current Super-Kamiokande detector (50 kton). With an alternative plan of locating one of the two identical detectors to Korea, better physics sensitivities are expected because of less muon flux and its spallation isotopes due to more overburden in Korean candidate sites than Japanese Hyper-Kamiokande site (Tochibora). According to our study using simple MC $102 \mathrm{SRN}$ events (5.2 sigma) in a Korean site and 71 SRN events (4.2 sigma) in the Japanese site are expected for 10 years of operation for one detector. Sensitivity studies using a full MC will be performed in the near future.

35th International Cosmic Ray Conference - ICRC2017

10-20 July, 2017

Bexco, Busan, Korea

\footnotetext{
*Speaker.
} 


\section{Hyper-Kamiokande}

Hyper-Kamiokande (Hyper-K or HK) is a next-generation, large-scale water Cherenkov neutrino detector which is planned to be built in Japan, as a successor of Super-Kamiokande (Super-K or SK). Hyper-Kamiokande consists of two identical cylindrical-shape $(60 \mathrm{~m}$ height $\times 74 \mathrm{~m}$ diameter) detectors with $40 \%$ photocoverage (40,000 photomultiplier tubes per tank). Each tank contains 260 kton purified water corresponding to 20 times larger fiducial volume $(187 \mathrm{kton} \times 2$ tanks) than that of SK. An alternative plan to locate one of the two tanks to a Korean site is called the second Hyper-K detector in Korea(HKK).
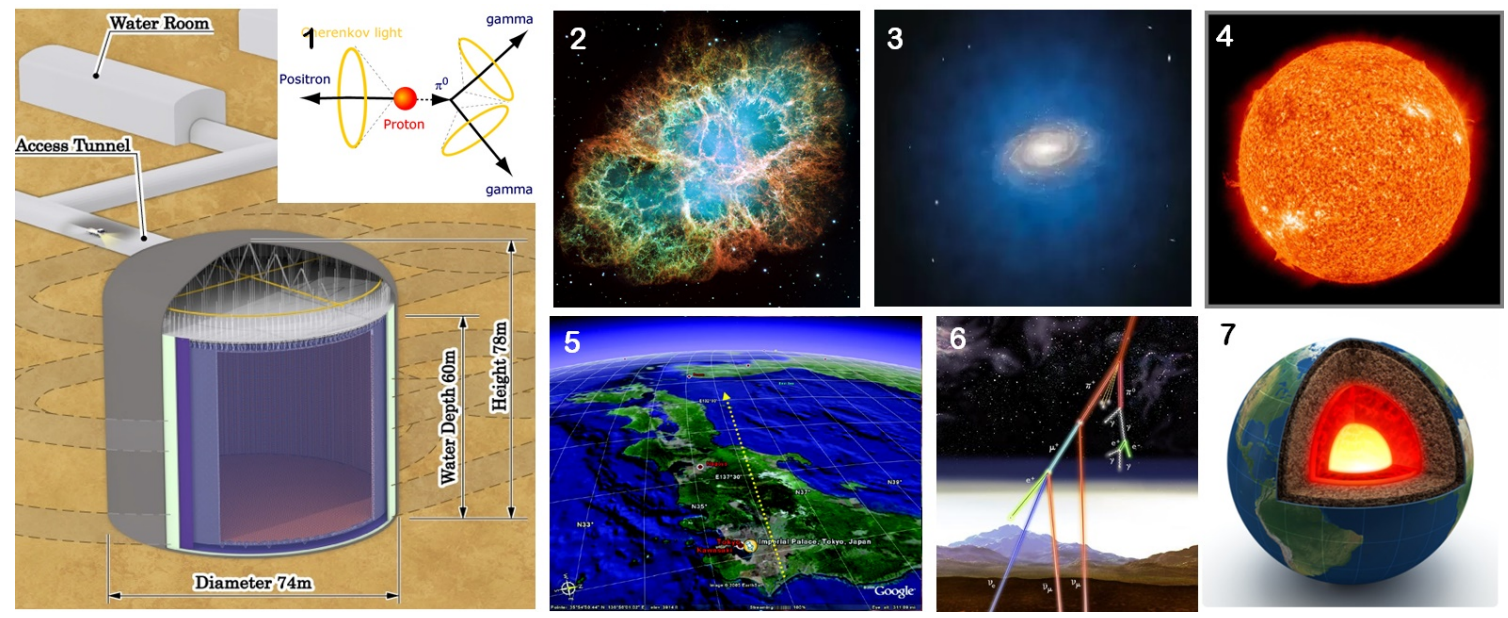

Figure 1: Hyper-K detector (bottom left) and its physics program. From left to right, above to below, are (1) proton decay search, (2) supernova burst and relic neutrino search, (3) dark matter search, (4) solar neutrino physics, (5) beam neutrino physics for CP violation phase measurement and neutrino mass ordering determination, (6) atmospheric neutrino physics, and (7) neutrino geophysics to explore the earth's interior.

Hyper-Kamiokande is a multi-purpose detector from beam neutrino physics for leptonic CP violation phase measurement and neutrino mass ordering determination to astrophysics, such as solar, supernova burst/relic neutrino physics, dark matter search, neutrino geophysics and proton decay.[1][2]. If one of the two tanks is located in Korea, the corresponding physics sensitivities are improved. First of all, for the beam neutrino physics, much longer baseline in Korea will break degeneracies related to the unknown neutrino mass ordering and the CP-violation in a leptonic sector. It will provide the opportunity to probe the oscillation of neutrinos, and reduce systematic uncertainties of the measurements. For the astrophysics, the atmospheric muon backgrounds are reduced due to a larger overburden in Korean sites, and therefore better physics sensitivities are expected. In this work, we focus on astrophysics especially on supernova burst and supernova relic neutrinos.

\section{Supernova Burst and SRN Motivation}

For massive stars $\left(>8 M_{\text {sun }}\right)$, their iron cores are formed at the end of the fusion process. Once an iron core is formed, then no more energy can be released from the fusion process, and the 
hydrodynamic balance between gravity and stellar burning is disrupted. This sudden instability of energy balance leads to a collapse of the core and as a result a huge explosion occurs. This is called a supernova burst. Because supernova emits $99 \%$ of its energy via neutrinos from its core, it is important to detect supernova neutrinos for understanding core collapse mechanism of supernovae. Another aspect of supernova-related neutrinos is supernova relic neutrinos, which are the ones from supernova burst in the past since the early universe. These two kinds of neutrinos are the main subjects of the supernova physics related to neutrinos. The detection of neutrinos from SN1987a indeed opens up a wide window to neutrino astronomy.

It is found that the main scenario of supernova burst is correct based on the three decades of supernova detection. However the process of core collapse supernova explosion mechanism is not revealed yet. The one of the strongest candidates of the core collapse supernova explosion mechanism is Standing Accretion Shock Instability (SASI)[3], a hydrodynamic instability of a halted shock wave in supernova cores. Fig. 2 (a) shows the SASI oscillation in a HK detector. Another example of an unsolved problem is the supernova rate problem (See Figure 2 (b)). According to star formation rate (SFR) in the history of our universe[4], the supernova rate is expected to be higher than observed supernova rate as a function of redshift. Observing SRN could solve this problem by their spectral shapes. Detection of supernova burst/relic neutrinos is the key to prove and understand the secrets of supernova.

Detection of SN neutrinos can be used as an alarm for the other astronomical optical telescopes, too. Supernova burst emits neutrinos earlier than photons because photons interact more with matters by electromagnetic interaction while neutrinos travels free due to their weak interactions. Depending on situations, neutrinos can arrive earlier by several hours to more than a day. Using a Hyper-K detector, the directionality of supernova burst is estimated as about 2 degree at 10 kpc (See Figure 2 (c)). This shows the possibility of SuperNova Early Warning System (SNEWS). The goal of the SNEWS is to provide prompt information of a supernova explosion to the astronomy community[5].

The HK in the Japanese site, Tochibora, is expected to detect 52,000-79,000 neutrinos events per tank for the supernova explosion occurring halfway across our galaxy[2]. It is about $5 \sim 8$ times more than that of SK[6]. With the much increased statistics it is expected to solve the supernova related problems with using HK. These improvements will open a new chapter for the future of supernova astronomy.

\section{Astrophysical Benefits of Korean Sites}

The overburden of the Korean candidate sites, such as Mt. Bisul or Mt. Bohyun, is $\sim 1 \mathrm{~km}$ while that of the Japanese HK site, Tochibora, is $650 \mathrm{~m}$. Therefore the atmospheric muon flux and its spallation isotopes are expected to be less in the Korean candidate sites as shown in Fig. 3 (a). This means, Korean sites have advantages on supernova burst/relic neutrino detection because of the low background rates leading to better physics sensitivities. Figure 3 (b) and (c) show side views of the Mt. Bisul and Mt. Bohyun with a tunnel length of $2.8 \mathrm{~km}$ and $3.9 \mathrm{~km}$, respectively. With a flat tunnel, the overburden becomes $\sim 820 \mathrm{~m}$ and therefore a tunnel in our consideration is sloped one to have a $\sim 1000$ m overburden. A geographical separation between HK and HKK can be another benefit to detect supernova burst neutrinos. 


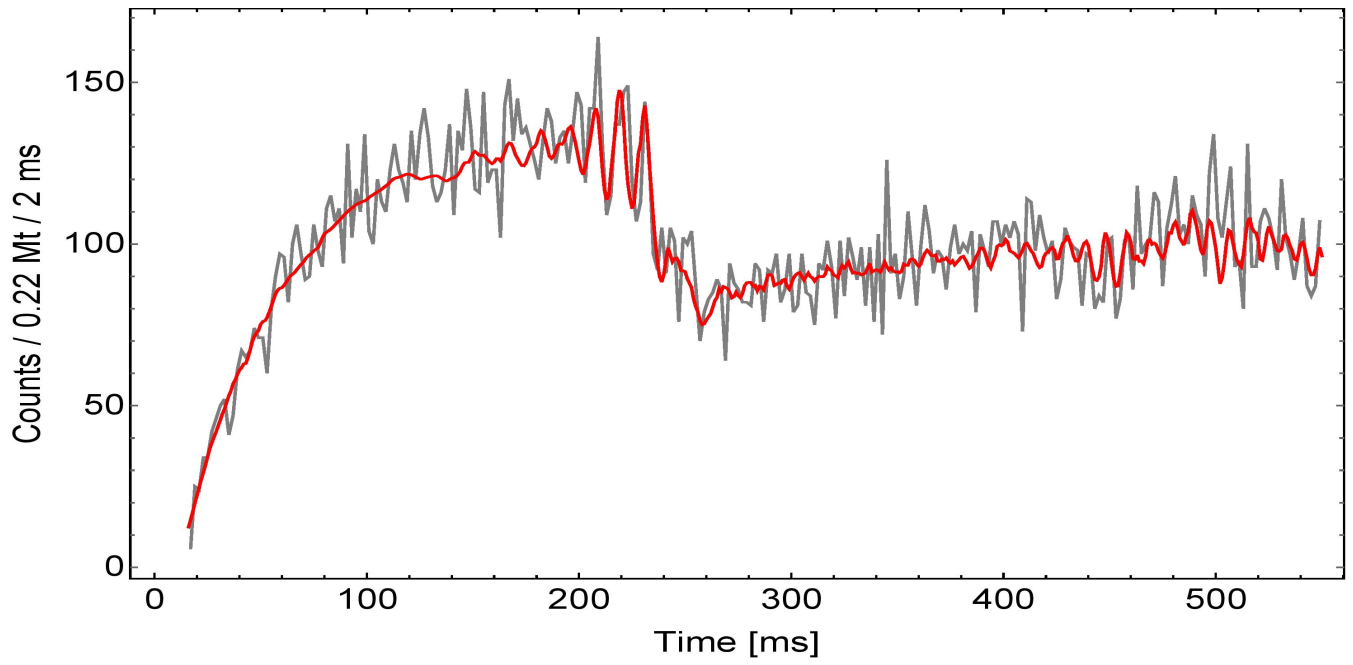

(a)

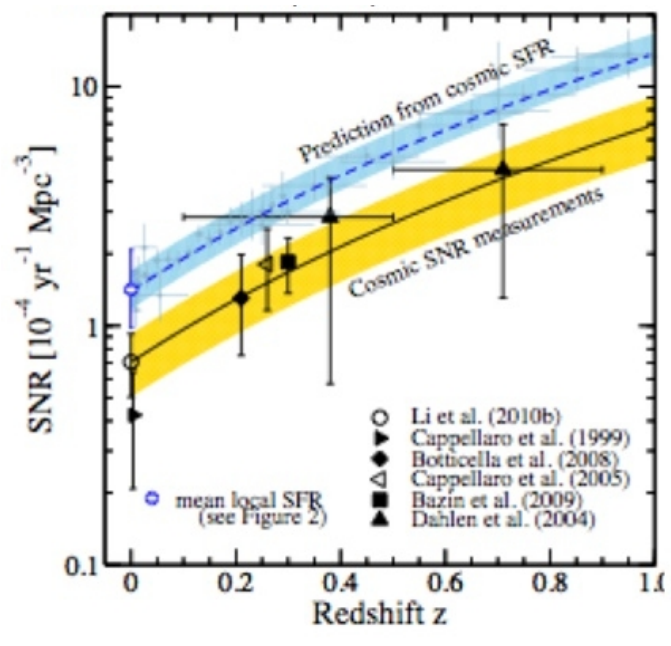

(b)

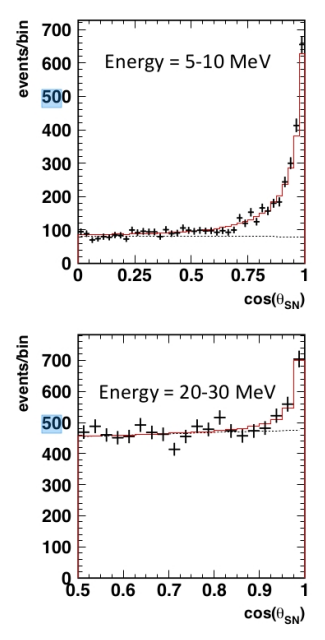

(c)
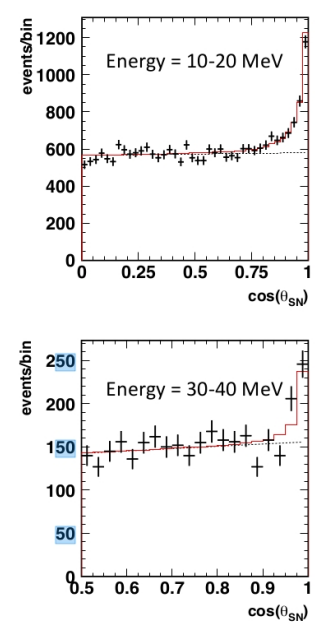

Figure 2: (a) Detected event rate modulation of SASI oscillation in HK 1 tank, where the progenitor mass is $27 M_{\text {sun }}$. Red line represents the theoretical event rate estimation for the inverse beta reaction and gray line represents for the event rate with statistical errors in $2 \mathrm{~ms}$ bins. (b) Supernova rate problem. Predicted supernova burst from the cosmic star formation history is plotted by dotted line with blue shadow, while the real detection of supernova burst is plotted by error bars with yellow shadow.[4] (c) Simulated angular distribution of a supernova burst at $10 \mathrm{kpc}$ with 2 tanks, for no oscillation case. The black dotted line and the red solid histogram (above the black dotted line) are fitted for the inverse beta decay and ve-scattering events, respectively.

\section{Supernova Burst and Relic Neutrinos in Japanese Site}

A simple MC study on supernova burst/relic neutrino detection is performed for a 10 -year operation of the staged 1 tank in Japanese site. In this detection scenario, for the case of SN at the galactic center (10 kpc), about (1) 49,000 to 68,000 supernova burst events from inverse beta decay(IBD), (2) 2,100 to 2,500 from ve-scattering, (3) 6 to 40 from neutronization, (4) 80 to 


\begin{tabular}{|c|c|c|}
\hline & Mt. Bisul & Mt. Bohyun \\
\hline Lattitude & $35^{\circ} 43^{\prime} 00^{\prime \prime} \mathrm{N}$ & $36^{\circ} 09^{\prime} 47^{\prime \prime} \mathrm{N}$ \\
\hline Longtitude & $128^{\circ} 31^{\prime} 28^{\prime \prime} \mathrm{E}$ & $128^{\circ} 58^{\prime} 26^{\prime} \mathrm{E}$ \\
\hline Tunnel Altitude (820 m overburden) & $264 \mathrm{~m}$ & $304 \mathrm{~m}$ \\
\hline Tunnel Altitude (1000m overburden) & $84 \mathrm{~m}$ & $124 \mathrm{~m}$ \\
\hline
\end{tabular}

Table 1: Location and the possible tunnel altitude of candidate sites in Korea. We can construct a detector at the deeper site than the HK in Japan for both candidate sites.

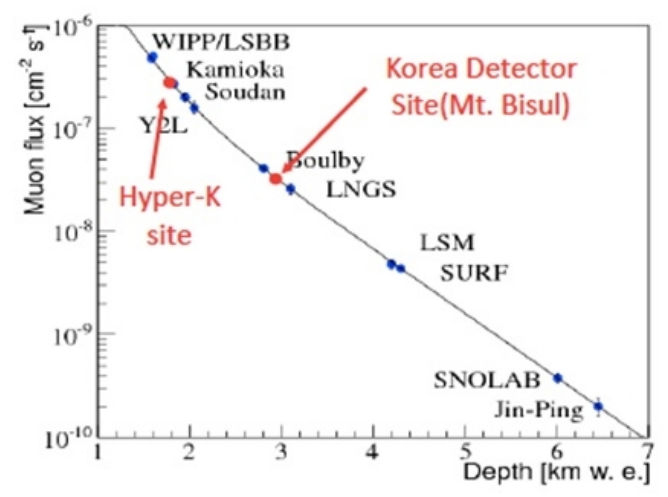

(a)

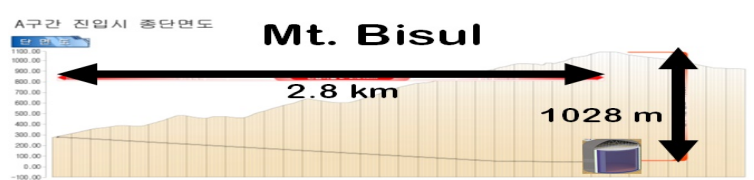

(b)

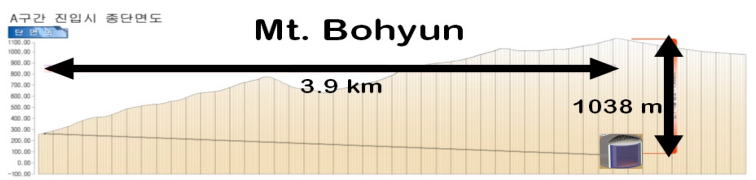

(c)

Figure 3: (a) Muon flux by depth in [km w.e.]. Dots represent specific experiment. The muon flux of Korean detector site(Mt. Bisul) is less than that of Hyper-K in Japan. (b) Mt. Bisul, with a 2.8 $\mathrm{km}$ tunnel length and $1028 \mathrm{~m}$ overburden. (c) Mt. Bohyun with a $3.9 \mathrm{~km}$ tunnel length and $1038 \mathrm{~m}$ overburden.

4,100 from $v_{e}$ charged current(CC) interaction, and (5) 650 to 3,900 from $\bar{v}_{e}$ CC interaction are expected(Figure 4 (a)). Figure 4 (b) shows the number of SRN is expected to be about 71 with significance of $4.1 \sigma$ for HK for 1 tank in Japan. It is not decided yet to add gadolinium to the HK detector, but the SRN search will be significantly improved if gadolinium is added (Figure 4 (c)).

For the Korean candidate sites, the sensitivity study on supernova burst neutrinos is still ongoing. The sensitivity study on SRN is described in the following section.

\section{SRN Sensitivity Studies at Korean sites}

Most important backgrounds of the SRN are the atmospheric muons and their spallation isotopes. We have simulated the muon flux and the spallation isotopes for the Korean candidate sites. Using a simple MC, the signal efficiency of SRN is estimated after applying background removal criteria.

\subsection{Simulation of Atmospheric Muon and Spallation Isotopes}

Before the estimation of the signal efficiency for SRN, it is a prerequisite to simulate the 


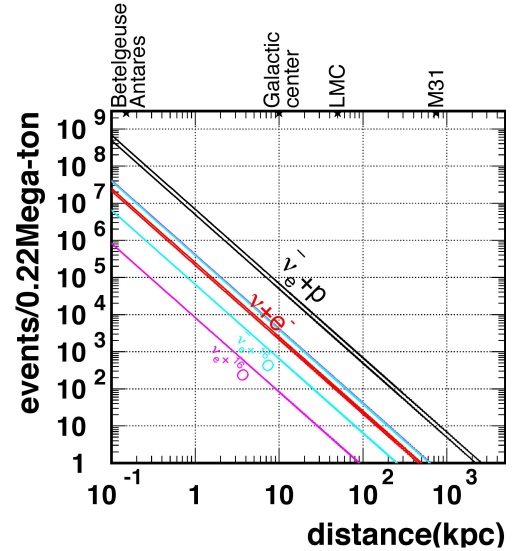

(a)

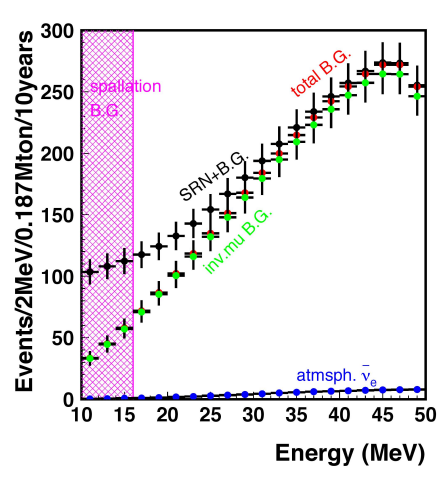

(b)

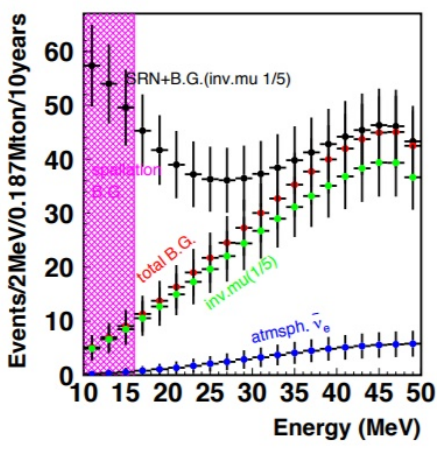

(c)

Figure 4: (a) Expected number of supernova burst events for each interaction as a function of the distance to a supernova for HK 1 tank. The band of each line shows the possible variation by the assumption of neutrino oscillations. (b) Expected spectrum of SRN signals at HK with 10 years of operation without tagging neutrons and (c) with gadolinium. Both plots are drawn for HK 1 tank assuming signal selection efficiency of $90 \%$. The black dots are the signal+total background, while the red dots are the total background. Green and blue dots are the invisible muon backgrounds and atmospheric $v_{e}$ components.

atmospheric muon flux and its spallation isotope yields. The softwares used in this study are MUSIC (MUon SImulation Code) for the muon flux and FLUKA for the spallation isotope yields, respectively. MUSIC is the $3 \mathrm{D}$ MC tool dedicated to muon transportation in matter. MUSIC takes into account the following muon energy loss mechanisms: (1) minimum ionization (Bethe-Bloch formula), (2) bremsstrahlung, (3) e-/e+ pair production, (4) muon-nucleus inelastic scattering[7]. FLUKA is a MC tool for particle transportation and their spallation interactions[8].

After simulating the muon flux and spallation isotope yields, the muon flux of the HK, SK, Mt. Bisul and Mt. Bohyun are compared. Backgrounds for two different depths, $820 \mathrm{~m}$ and 1 $\mathrm{km}$, are simulated for both Mt. Bisul and Mt. Bohyun. If the overburden is $820 \mathrm{~m}$, the muon flux rate and the spallation rate for the Korean sites are higher than that of SK site. For the Mt. Bisul, the muon flux is estimated as $2.47 \pm 0.49$ times higher and ${ }^{16} \mathrm{~N}$ rate is estimated as $1.99 \pm 0.44$ times higher than that of SK. However, for the $1 \mathrm{~km}$ depth of the Mt. Bisul, the backgrounds are reduced to $1.03 \pm 0.21$ and $\sim 1$ times, respectively, i.e. comparable amount of backgrounds in SK. On the other hand, muon flux of the Japanese HK site is about $4.90 \pm 0.98$ times higher than that of SK. Therefore, the backgrounds of Korean sites are expected to be much less than that of the Japanese HK site. Figure 5 shows muon flux as a function of zenith and azimuthal angle (East, West, North, and South) for $820 \mathrm{~m}$ and $1000 \mathrm{~m}$ overburdens of Mt. Bisul and Mt. Bohyun where the muon fluxes of SK and the Japanese HK sites are overlaid for comparisons. The background muon flux/spallation isotopes in the HK detectors are estimated using the following relation:

$$
\operatorname{Rate}(H K)=\operatorname{Rate}(S K) \times \frac{\phi(H K)}{\phi(S K)} \times \frac{Y(H K)}{Y(S K)} .
$$




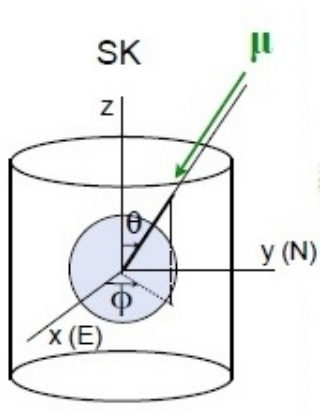

(a)

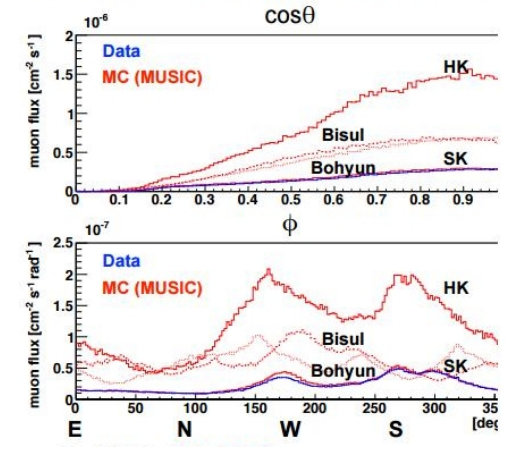

(b)
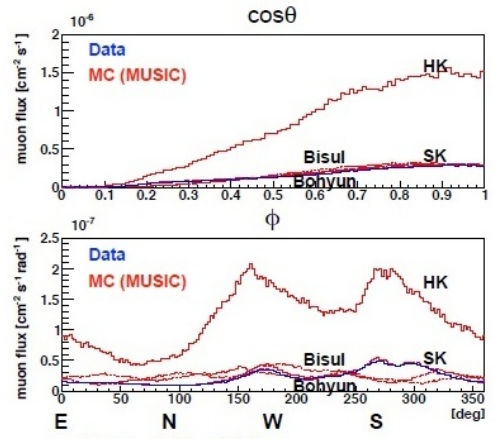

(c)

Figure 5: (a) Schematic view of muon backgrounds simulation. Muon flux measured by 12 days data in spherical volume or $\mathrm{R}=10 \mathrm{~m}$. (b) Comparison of muon flux for the HK/SK/Mt. Bisul/Mt. Bohyun for the $820 \mathrm{~m}$ overburden. (c) Comparison of muon flux for the HK/SK/Mt. Bisul/Mt. Bohyun for the $1000 \mathrm{~m}$ overburden. For both plots (b) and (c), top plots are the muon flux by the zenith angle, while the bottom plots are the muon flux by the azimuthal angle.

\subsection{Signal Efficiency for SRN search}

After the background simulation, SRN signal events are generated using a simple MC. The signal efficiency for SRN is estimated by applying selection criteria to remove the backgrounds[9]. Figure 6 (a) shows the number of SRN events over operation years and (b) shows the corresponding significances of SRN detection in the Japanese and Korean candidate sites. According to this study as listed in Table 2, it is expected that 102 SRN events $(5.2 \sigma)$ in the Korean Mt. Bisul (1 km depth) site while 71 SRN events $(4.2 \sigma)$ in the Japanese site for 10 years of operation with one tank.

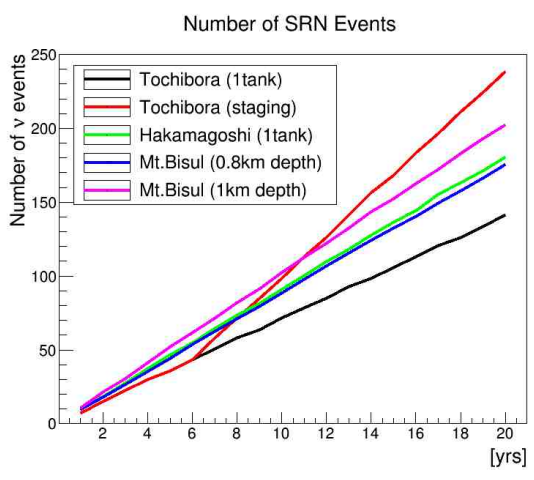

(a)

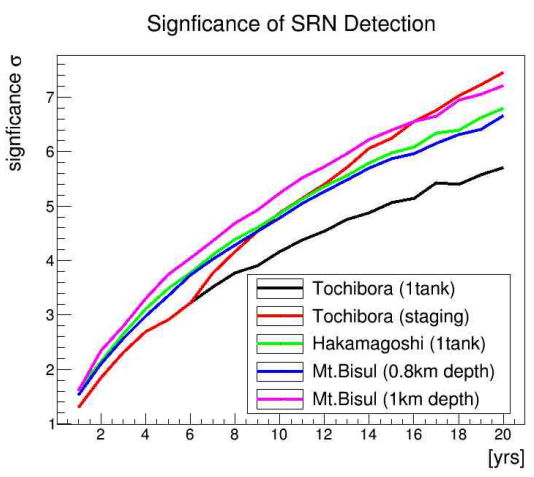

(b)

Figure 6: (a) Expected number of SRN events over time for different locations. For the one tank experiments, Mt. Bisul of $1 \mathrm{~km}$ overburden is the best site to detect SRN events. (b) Expected significance over time. For the one tank experiments, significance of Mt. Bisul with $1 \mathrm{~km}$ overburden is the best among them. 


\begin{tabular}{|c|c|c|c|c|}
\hline Location & No. of Tank & Depth $(\mathrm{km})$ & No. of Events $(/ 10 \mathrm{yrs})$ & Significance $\sigma(/ 10 \mathrm{yrs})$ \\
\hline Tochibora & 2 , staging & 0.65 & $\sim 98$ & $\sim 4.9$ \\
\hline Tochibora* & 1 & 0.65 & $\sim 71$ & $\sim 4.2$ \\
\hline Hakamagoshi & 1 & 0.85 & $\sim 91$ & $\sim 4.9$ \\
\hline Mt. Bisul & 1 & 0.82 & $\sim 88$ & $\sim 4.8$ \\
\hline Mt. Bisul* & 1 & 1 & $\sim 102$ & $\sim 5.2$ \\
\hline
\end{tabular}

Table 2: Expected number of events/significance for the 5 different sites of Korea and Japan. Note that in the Tochibora (one tank) and Mt. Bisul with $1 \mathrm{~km}$ depth, the number of detected events, 71 vs. 102 , and significance, $4.2 \sigma$ vs. $5.2 \sigma$, are higher in the Mt. Bisul.

\section{Summary}

We have simulated atmospheric muon and its spallation isotope backgrounds at the Korean candidate sites, and estimated SRN sensitivity using a simple MC. If one of two Hyper-K tanks is located in the Korean candidate sites, sensitivities on supernova burst and supernova relic neutrinos are expected to be improved with less muon backgrounds thanks to the larger overburden of the Korean candidates. Results with a full MC simulation will be performed soon.

\section{References}

[1] Hyper-Kamiokande Proto-Collaboration, Physics Potentials with the Second Hyper-Kamiokande Detector in Korea, [1611.06118] (2016).

[2] Hyper-Kamiokande collaboration, Hyper-Kamiokande Design Report, KEK-PREPRINT-2016-21, ICRR-REPORT-701-2016-1 (2016).

[3] Tomoya Takiwaki, Kei Kotake, and Yudai Suwa , A comparison of two- and three-dimensional neutrino-hydrodynamics simulations of core-collapse supernovae, Astrophys.J.786 (2014) 83-90, [1308.5755].

[4] S.Horiuchi et al., The cosmic core-collapse supernovva rate does not match the massive-star formation rate, Astrophys.J. 738 (2011) 154-169, [1102 . 1977].

[5] P. Antonioli et al, SNEWS: The SuperNova Early Warning System, New J. Phys. 6 (2004) 114, [astro-ph/0406214].

[6] The Super-Kamiokande Collaboration, Search for supernova neutrino burst at Super-Kamiokande, Astrophys.J. 669 (2007) 519-524, [0 706 .2283].

[7] V. A. Kudryavtsev, Muon simulation codes MUSIC and MUSUN for underground physics, [0810.4635] (2008).

[8] A. Ferrari, P. R. Sala, A. Fasso, and J. Ranft, FLUKA: A multi-particle transport code, INFN-TC-05-11, SLAC-R-773, CERN-2005-10 (2005).

[9] Kirk Bays et al., Supernova relic neutrino search at super-Kamiokande, Phys.Rev. D.85 (2012) 052007, [1111.5031]. 